Pré-Publicações do Departamento de Matemática

Universidade de Coimbra

Preprint Number 09-30

\title{
THE SEMICONTINUOUS QUASI-UNIFORMITY OF A FRAME, REVISITED
}

\author{
MARIA JOÃO FERREIRA AND JORGE PICADO
}

\begin{abstract}
In this note we present a new treatment of the pointfree version of the semicontinuous quasi-uniformity based on the new tool of the ring of arbitrary (not necessarily continuous) real-valued functions made available recently by J. Gutiérrez García, T. Kubiak and J. Picado [Localic real functions: a general setting, Journal of Pure and Applied Algebra 213 (2009) 1064-1074]. The purpose is to show how the basic facts about the semicontinuous quasi-uniformity can be easily presented and proved with that tool at hand.

KEYWORDS: Frame, quasi-uniform frame, quasi-uniform biframe, quasi-metric quasiuniformity, totally bounded quasi-uniformity, semicontinuous real function, biframe of reals, countably compact frame.
\end{abstract}

AMS Subject Classification (2000): 06D22, 54C30, 54E05, 54E15, 54E55.

\section{Introduction}

Let $X$ be a locale with corresponding frame $L=\mathcal{O}(X)$. The lattice of sublocales of $X$ (that is, the subobject lattice of $X$ in the category of locales) may be described in several equivalent ways. Here we use the following one [18]:

a subset $S$ of $L$ is a sublocale of $X$ if, whenever $A \subseteq S, a \in L$ and $b \in S$, then $\bigwedge A \in S$ and $a \rightarrow b \in S$.

Any intersection of sublocales is again a sublocale, so that the set of all sublocales is a complete lattice under inclusion. In fact, it is a co-frame. We make it into a frame $\mathcal{S}(L)$ by considering the dual ordering $S_{1} \leq S_{2}$ iff $S_{2} \subseteq S_{1}$. Among the important examples of sublocales are the closed sublocales

$$
\mathfrak{c}(a)=\uparrow a=\{b \in L: a \leq b\}
$$

Received August 16, 2009.

The authors gratefully acknowledge financial support by the Centre for Mathematics of the University of Coimbra (CMUC/FCT). The second named author also acknowledges support from the Ministry of Science and Innovation of Spain and FEDER under grant MTM2009-12872-C02-02. 
and the open sublocales

$$
\mathfrak{o}(a)=\{a \rightarrow b: b \in L\}
$$

for every $a \in L$ (which are complements of each other). The map $a \mapsto \mathfrak{c}(a)$ is a frame embedding $L \hookrightarrow \mathcal{S}(L)$. The subframe of $\mathcal{S}(L)$ consisting of all closed sublocales will be denoted by $\mathfrak{c} L$. It is isomorphic to $L$. Denoting by $\mathfrak{o} L$ the subframe of $\mathcal{S}(L)$ generated by all $\mathfrak{o}(a), a \in L$, the triple $(\mathcal{S}(L), \mathfrak{c} L, \mathfrak{o} L)$ constitutes a biframe.

It is well-known that a quasi-uniformity $\mathcal{E}$ on a set $X$ may be described in several equivalent ways, most notably as a collection of ordered pairs of covers of $X$ (the paircover approach) and as a collection of relations on $X$ (the entourage approach). Associated with any quasi-uniformity $\mathcal{E}$ on $X$ there is the bitopological space $\left(X, \mathfrak{T}_{\mathcal{E}}, \mathfrak{T}_{\mathcal{E}^{-1}}\right)$ induced by $\mathcal{E}$.

In the pointfree setting, the theory of quasi-uniformities was first exploited using the paircover approach [8, 9]; the Weil entourages of $[15,16,17]$ provided then the direct analogue of entourages. The former is defined as a structure $\mathcal{U}$ on a biframe $\left(L_{0}, L_{1}, L_{2}\right)$ and the latter directly as a structure $\mathcal{E}$ on a frame $L$ which establishes two subframes $L_{1}(\mathcal{E})$ and $L_{2}(\mathcal{E})$ of $L$ such that the triple $\left(L, L_{1}(\mathcal{E}), L_{2}(\mathcal{E})\right)$ is a biframe (this is the pointfree version of the bitopological space $\left(X, \mathfrak{T}_{\mathcal{E}}, \mathfrak{T}_{\mathcal{E}^{-1}}\right)$ above). The two approaches are equivalent $[15,16]$.

While the approach via paircovers is most convenient for calculations (the entourage approach asks for a good knowledge of the construction of binary coproducts of frames), the entourage approach allows to formulate the theory directly on frames, in a way very similar to the spatial setting $[4,5,17]$. For instance, given a frame $L$, there exists a (entourage) transitive quasiuniformity $\mathcal{E}$ on the sublocale frame $\mathcal{S}(L)$ which is compatible with $L$, that is, $L_{1}(\mathcal{E})=\mathfrak{c} L$ (which means that $L_{1}(\mathcal{E})$ is an isomorphic copy of the given frame $L$ inside $\mathcal{S}(L))[4,5]$. This is the pointfree analogue of the well-known classical fact that for every topological space $(X, \mathfrak{T})$ there exists a transitive quasi-uniformity $\mathcal{E}$ on $X$, compatible with $(X, \mathfrak{T})$, that is, which induces as its first topology $\mathfrak{T}_{\mathcal{E}}$ the given topology $\mathfrak{T}$.

The semicontinuous quasi-uniformity $\mathcal{U S C}(L)$ of $L$ is a nice example of a transitive compatible quasi-uniformity $[5,6]$. The purpose of this paper is to show how the basic facts about $\mathcal{U S C}(L)$ can be nicely presented with the help of the ring of arbitrary (not necessarily continuous) real-valued functions made available recently by J. Gutiérrez García, T. Kubiak and J. Picado 
[12]. To keep the background at the minimum possible we use the paircover approach $[8,10]$ to quasi-uniformities.

\section{Background}

For general information on locales and frames we refer to [13] and [18]. A biframe [2] is a triple $L=\left(L_{0}, L_{1}, L_{2}\right)$ in which $L_{0}$ is a frame, $L_{1}$ and $L_{2}$ are subframes of $L_{0}$ and $L_{1} \cup L_{2}$ generates $L_{0}$ (by joins of finite meets). A biframe map $h: L \rightarrow M$ is a frame homomorphism from $L_{0}$ to $M_{0}$ such that the image of $L_{i}$ under $h$ is contained in $M_{i}$ for $i=1,2$. Biframes and biframe maps are the objects and arrows of the category BiFrm. For more details on biframes consult [2].

Let $L=\left(L_{0}, L_{1}, L_{2}\right)$ be a biframe. A subset $C$ of $L_{1} \times L_{2}$ is a paircover $[8,10]$ of $L$ if $\bigvee\left\{c_{1} \wedge c_{2} \mid\left(c_{1}, c_{2}\right) \in C\right\}=1$. A paircover $C$ of $L$ is strong if, for any $\left(c_{1}, c_{2}\right) \in C, c_{1} \vee c_{2}=0$ whenever $c_{1} \wedge c_{2}=0$ (that is, $\left(c_{1}, c_{2}\right)=(0,0)$ whenever $\left.c_{1} \wedge c_{2}=0\right)$.

For any paircovers $C$ and $D$ of $L$ we write $C \leq D$ (and say that $C$ refines $D)$ if for any $\left(c_{1}, c_{2}\right) \in C$ there is $\left(d_{1}, d_{2}\right) \in D$ with $c_{1} \leq d_{1}$ and $c_{2} \leq d_{2}$. Further $C \wedge D=\left\{\left(c_{1} \wedge d_{1}, c_{2} \wedge d_{2}\right) \mid\left(c_{1}, c_{2}\right) \in C,\left(d_{1}, d_{2}\right) \in D\right\}$. It is obvious that $C \wedge D$ is a paircover of $L$. For $a \in L_{0}$ and $C, D$ paircovers of $L$, let

$$
\begin{aligned}
& s t_{1}(a, C)=\bigvee\left\{c_{1} \mid\left(c_{1}, c_{2}\right) \in C \text { and } c_{2} \wedge a \neq 0\right\}, \\
& s t_{2}(a, C)=\bigvee\left\{c_{2} \mid\left(c_{1}, c_{2}\right) \in C \text { and } c_{1} \wedge a \neq 0\right\}
\end{aligned}
$$

and

$$
C \cdot D=\left\{\left(s t_{1}\left(d_{1}, C\right), s t_{2}\left(d_{2}, C\right)\right) \mid\left(d_{1}, d_{2}\right) \in D\right\} .
$$

The particular case $C \cdot C$ is usually denoted by $C^{*}$. The paircover $C$ is said to star-refines $D$ if $C^{*} \leq D$.

The following lemma is easy to prove [8].

Lemma 2.1. For any paircovers $C, D$ of $\left(L_{0}, L_{1}, L_{2}\right)$ and any $a, b \in L_{0}$ we have:

(1) $a \leq s t_{i}(a, C)(i=1,2)$.

(2) $a \leq b \Rightarrow s t_{i}(a, C) \leq s t_{i}(b, C)(i=1,2)$.

(3) If $D^{*} \leq C$ then $s t_{i}\left(s t_{i}(a, D), D\right) \leq s t_{i}(a, C)(i=1,2)$.

(4) For any biframe map $h:\left(L_{0}, L_{1}, L_{2}\right) \rightarrow\left(M_{0}, M_{1}, M_{2}\right)$, st $t_{i}(h(a), h[C]) \leq$ $h\left(\operatorname{st}_{i}(a, C)\right)(i=1,2)$, where $h[C]=\left\{\left(h\left(c_{1}\right), h\left(c_{2}\right)\right) \mid\left(c_{1}, c_{2}\right) \in C\right\}$. 
A non-empty family $\mathcal{U}$ of paircovers of $L=\left(L_{0}, L_{1}, L_{2}\right)$ is a quasi-uniformity on $L$ if:

(U1) The family of strong members of $\mathcal{U}$ is a filter-base for $\mathcal{U}$ with respect to $\wedge$ and $\leq$.

(U2) For any $C \in \mathcal{U}$ there is $D \in \mathcal{U}$ such that $D^{*} \leq C$.

(U3) For each $a \in L_{i}, a=\bigvee\left\{b \in L_{i} \mid s t_{i}(b, C) \leq a\right.$ for some $\left.C \in \mathcal{U}\right\}$, $(i=1,2)$.

The pair $(L, \mathcal{U})$ is called a quasi-uniform biframe [10]. $\mathcal{B} \subseteq \mathcal{U}$ is a base for $\mathcal{U}$ if, for each $C \in \mathcal{U}$, there is $B \in \mathcal{B}$ such that $B \leq C$.

Let $(L, \mathcal{U})$ and $(M, \mathcal{V})$ be quasi-uniform biframes. A biframe map $h: L \rightarrow$ $M$ is uniform if for every $C \in \mathcal{U}, h[C] \in \mathcal{V}$. Quasi-uniform biframes and uniform maps constitute a category that we denote by QUBiFrm.

The biframe of reals is the triple $\left(\mathfrak{L}(\mathbb{R}), \mathfrak{L}_{l}(\mathbb{R}), \mathfrak{L}_{u}(\mathbb{R})\right)$ where $\mathfrak{L}(\mathbb{R})$ is the frame of reals $[1]$ defined by generators $(p, q) \in \mathbb{Q} \times \mathbb{Q}$ and relations

(R1) $(p, q) \wedge(r, s)=(p \vee r, q \wedge s)$,

(R2) $(p, q) \vee(r, s)=(p, s)$ whenever $p \leq r<q \leq s$,

(R3) $(p, q)=\bigvee\{(r, s): p<r<s<q\}$,

(R4) $\bigvee_{p, q \in \mathbb{Q}}(p, q)=1$.

We shall use also the following notation:

$$
(p,-)=\bigvee_{q \in \mathbb{Q}}(p, q) \quad \text { and }(-, q)=\bigvee_{p \in \mathbb{Q}}(p, q) ;
$$

note that $(p,-) \wedge(-, q)=(p, q)$.

Equivalently, $\mathfrak{L}(\mathbb{R})$ may be defined by taking $(p,-)$ and $(-, q)$ as primitive notions, with relations

(S1) $(p,-) \wedge(-, q)=0$ whenever $p \geq q$,

(S2) $(p,-) \vee(-, q)=1$ whenever $p<q$,

(S3) $(p,-)=\bigvee_{r>p}(r,-)$,

(S4) $(-, q)=\bigvee_{s<q}(-, s)$,

(S5) $\bigvee_{p \in \mathbb{Q}}(p,-)=1$

(S6) $\bigvee_{q \in \mathbb{Q}}(-, q)=1$.

Then $\mathfrak{L}_{u}(\mathbb{R})$ and $\mathfrak{L}_{l}(\mathbb{R})$ are just the following subframes of $\mathfrak{L}(\mathbb{R})$ :

$\mathfrak{L}_{u}(\mathbb{R})=\langle\{(p,-): p \in \mathbb{Q},(p,-)$ satisfy $(\mathrm{R} 3)$ and (R5) for all $p \in \mathbb{Q}\}\rangle$,

$\mathfrak{L}_{l}(\mathbb{R})=\langle\{(-, q): q \in \mathbb{Q},(-, q)$ satisfy $(\mathrm{R} 4)$ and $(\mathrm{R} 6)$ for all $q \in \mathbb{Q}\}\rangle$. 
In general topology one sometimes deals with arbitrary (not necessarily continuous) real-valued functions on a topological space $X$. This is also possible in the pointfree setting with the approach recently introduced in [12] (which extends the approach to pointfree continuous real functions of Banaschewski [1]). Let $L$ be a frame. A real-valued function on $L$ is a frame homomorphism $f: \mathfrak{L}(\mathbb{R}) \rightarrow \mathcal{S}(L)$. It is

(1) lower semicontinuous if $f\left(\mathfrak{L}_{u}(\mathbb{R})\right) \subseteq \mathfrak{c} L$,

(2) upper semicontinuous if $f\left(\mathfrak{L}_{l}(\mathbb{R})\right) \subseteq \mathfrak{c} L$,

(3) continuous if $f(\mathfrak{L}(\mathbb{R})) \subseteq \mathfrak{c} L$.

The set $\mathrm{F}(L)$ of all real-valued functions on $L$ is partially ordered by

$$
\begin{array}{rlll}
f \leq g & \Leftrightarrow \quad f(p,-) \leq g(p,-) & \text { for every } p \in \mathbb{Q} \\
& \Leftrightarrow \quad g(-, q) \leq f(-, q) & \text { for every } q \in \mathbb{Q} .
\end{array}
$$

We denote by $\operatorname{LSC}(L), \operatorname{USC}(L)$ and $\mathrm{C}(L)$ the collections of all lower semicontinuous, upper semicontinuous, and continuous members of $\mathrm{F}(L)$. Of course, one has

$$
\mathrm{C}(L)=\operatorname{LSC}(L) \cap \operatorname{USC}(L) .
$$

Note that $\operatorname{USC}(L) \simeq \operatorname{BiFrm}\left(\left(\mathfrak{L}(\mathbb{R}), \mathfrak{L}_{l}(\mathbb{R}), \mathfrak{L}_{u}(\mathbb{R})\right),(\mathcal{S}(L), \mathfrak{c} L, \mathfrak{o} L)\right)$.

A nice way of constructing real functions is with the help of the so called scales [12]. A collection of sublocales $\left\{S_{r}: r \in \mathbb{Q}\right\} \subseteq \mathcal{S}(L)$ is a scale on $\mathcal{S}(L)$ if $S_{r} \vee S_{s}^{*}=1$ whenever $r<s$ and $\bigvee\left\{S_{r}: r \in \mathbb{Q}\right\}=1=\bigvee\left\{S_{r}^{*}: r \in \mathbb{Q}\right\}$ (here $S^{*}$ denotes the pseudocomplement of $S$ ). For each scale $\left\{S_{r}: r \in \mathbb{Q}\right\}$ in $\mathcal{S}(L)$ the function $f$ defined by

$$
f(p,-)=\bigvee_{r>p} S_{r} \quad \text { and } \quad f(-, q)=\bigvee_{r<q} S_{r}{ }^{*} \quad(p, q \in \mathbb{Q})
$$

belongs to $\mathrm{F}(L)$. If, moreover, each $S_{r}$ is an open sublocale then $f \in \operatorname{USC}(L)$.

For instance, given a complemented sublocale $S$ of $L$, with complement $\neg S$, the characteristic map $\chi_{S}: \mathfrak{L}(\mathbb{R}) \rightarrow \mathcal{S}(L)$ is defined by

$$
\chi_{S}(p,-)=\left\{\begin{array}{ll}
1 & \text { if } p<0, \\
\neg S & \text { if } 0 \leq p<1, \\
0 & \text { if } p \geq 1,
\end{array} \quad \text { and } \quad \chi_{S}(-, q)= \begin{cases}0 & \text { if } q \leq 0, \\
S & \text { if } 0<q \leq 1 \\
1 & \text { if } q>1,\end{cases}\right.
$$

for each $p, q \in \mathbb{Q}[12]$. Then, as in the classical context, we have:

(a) $\chi_{S} \in \operatorname{LSC}(L)$ if and only if $S$ is open,

(b) $\chi_{S} \in \mathrm{USC}(L)$ if and only if $S$ is closed, 
(c) $\chi_{S} \in \mathrm{C}(L)$ if and only if $S$ is clopen.

For any $f \in \mathrm{F}(L)$ the upper regularization $f^{-} \in \operatorname{USC}(L)$ of $f$ is defined by

$$
f^{-}(p,-)=\bigvee_{q>p} \neg \overline{f(-, q)} \quad \text { and } \quad f^{-}(-, p)=\bigvee_{q<p} \overline{f(-, q)}
$$

(see [11] and [12] for more information). Of course, when $f \in \operatorname{USC}(L)$ then $f^{-}=f$. Thus, for any $f \in \mathrm{USC}(L)$, we have

$$
f(p,-)=\bigvee_{q>p} \neg f(-, q) \in \mathfrak{o} L \quad \text { and } \quad f(-, p)=\bigvee_{q<p} f(-, q) \in \mathfrak{c} L .
$$

\section{The semicontinuous quasi-uniformity $\mathcal{U S C}(L)$}

For each $n \in \mathbb{N}$,

$$
Q_{n}=\left\{((-, q),(p,-)) \mid p, q \in \mathbb{Q}, 0<q-p<\frac{1}{n}\right\}
$$

is a strong paircover of the biframe $\left(\mathfrak{L}(\mathbb{R}), \mathfrak{L}_{l}(\mathbb{R}), \mathfrak{L}_{u}(\mathbb{R})\right)$. These paircovers satisfy the following (easy to check) properties:

Lemma 3.1. (1) For every $n \in \mathbb{N}$ and $p, q \in \mathbb{Q}$ with $p<q, \frac{1}{q-p}<n$, we have:

(a) $s t_{1}\left((-, p), Q_{n}\right) \leq(-, q)$.

(b) $s t_{2}\left((q,-), Q_{n}\right) \leq(p,-)$.

(2) For every $p_{i}, q_{i} \in \mathbb{Q}$ with $p_{i}<q_{i}$, we have:

(a) $s t_{1}\left(\bigvee_{i \in I}\left(p_{i}, q_{i}\right), Q_{n}\right)=s t_{1}\left(\bigvee_{i \in I}\left(-, q_{i}\right), Q_{n}\right)$.

(b) $s t_{2}\left(\bigvee_{i \in I}\left(p_{i}, q_{i}\right), Q_{n}\right)=s t_{2}\left(\bigvee_{i \in I}\left(p_{i},-\right), Q_{n}\right)$.

(3) For each $n \in \mathbb{N}, Q_{n+1} \subseteq Q_{n}$ (thus $Q_{n+1} \leq Q_{n}$ ).

Moreover:

Proposition 3.2. For every $n \in \mathbb{N}$ and $p \in \mathbb{Q}$, we have:

(1) $Q_{3 n}^{*} \leq Q_{n}$.

(2) $(-, p)=\bigvee\left\{(-, q) \in \mathfrak{L}_{l}(\mathbb{R}) \mid s t_{1}\left((-, q), Q_{n}\right) \leq(-, p)\right.$ for some $\left.n \in \mathbb{N}\right\}$.

(3) $(p,-)=\bigvee\left\{(q,-) \in \mathfrak{L}_{u}(\mathbb{R}) \mid s_{2}\left((q,-), Q_{n}\right) \leq(p,-)\right.$ for some $n \in$ $\mathbb{N}\}$. 
Proof: (1) Let $((-, q),(p,-)) \in Q_{3 n}$. We have to show that there is

$$
((-, \tilde{q}),(\tilde{p},-)) \in Q_{n}
$$

such that $s t_{1}\left((-, q), Q_{3 n}\right) \leq(-, \tilde{q})$ and $s t_{2}\left((p,-), Q_{3 n}\right) \leq(\tilde{p},-)$. But

$$
\begin{aligned}
s t_{1}\left((-, q), Q_{3 n}\right) & =\bigvee\left\{\left(-, d_{1}\right) \mid\left(\left(-, d_{1}\right),\left(d_{2},-\right)\right) \in Q_{3 n},\left(d_{2},-\right) \wedge(-, q) \neq 0\right\} \\
& \leq\left(-, q+\frac{1}{3 n}\right)
\end{aligned}
$$

since $\left(d_{2},-\right) \wedge(-, q) \neq 0 \Leftrightarrow d_{2}<q$ and $0<d_{1}-d_{2}<\frac{1}{3 n}$ (which implies $\left.d_{1}<d_{2}+\frac{1}{3 n}<q+\frac{1}{3 n}\right)$. Similarly,

$$
\begin{aligned}
s t_{2}\left((p,-), Q_{3 n}\right) & =\bigvee\left\{\left(d_{2},-\right) \mid\left(\left(-, d_{1}\right),\left(d_{2},-\right)\right) \in Q_{3 n},\left(-, d_{1}\right) \wedge(p,-) \neq 0\right\} \\
& \leq\left(p-\frac{1}{3 n},-\right) .
\end{aligned}
$$

It suffices then to take $\tilde{q}=q+\frac{1}{3 n}$ and $\tilde{p}=p-\frac{1}{3 n}$. Indeed, $\left(\left(-, q+\frac{1}{3 n}\right),(p-\right.$ $\left.\left.\frac{1}{3 n},-\right)\right) \in Q_{n}$, since $0<q+\frac{1}{3 n}-p+\frac{1}{3 n}<\frac{1}{3 n}+\frac{1}{3 n}+\frac{1}{3 n}=\frac{1}{n}$.

(2) By Lemma 3.1(1), for every $q<p$ there is some $n \in \mathbb{N}$ such that $s t_{1}\left((-, q), Q_{n}\right) \leq(-, p)$. Thus, by Lemma 2.1(1),

$$
\begin{aligned}
(-, p) & =\bigvee_{q<p}(-, q) \leq \bigvee\left\{(-, q) \mid s t_{1}\left((-, q), Q_{n}\right) \leq(-, p) \text { for some } n \in \mathbb{N}\right\} \\
& \leq(-, p) .
\end{aligned}
$$

(3) may be proved similarly.

In conclusion, the strong paircovers $Q_{n}(n \in \mathbb{N})$, generate a quasi-uniformity $\mathcal{Q}$ on the biframe of reals $\left(\mathfrak{L}(\mathbb{R}), \mathfrak{L}_{l}(\mathbb{R}), \mathfrak{L}_{u}(\mathbb{R})\right)$.

Corollary 3.3. The pair $\left(\left(\mathfrak{L}(\mathbb{R}), \mathfrak{L}_{l}(\mathbb{R}), \mathfrak{L}_{u}(\mathbb{R})\right), \mathcal{Q}\right)$ is a quasi-uniform biframe.

We refer to it as the quasi-metric quasi-uniformity of the reals.

Now let $f \in \operatorname{USC}(L)$. Then (recall (2.2))

$$
f(p,-)=\bigvee_{q>p} \neg f(-, q) \in \mathfrak{o} L \quad \text { and } \quad f(-, p)=\bigvee_{q<p} f(-, q) \in \mathfrak{c} L
$$

so $f: \mathfrak{L}(\mathbb{R}) \rightarrow \mathcal{S}(L)$ is a biframe map

$$
f:\left(\mathfrak{L}(\mathbb{R}), \mathfrak{L}_{l}(\mathbb{R}), \mathfrak{L}_{u}(\mathbb{R})\right) \rightarrow(\mathcal{S}(L), \mathfrak{c} L, \mathfrak{o} L) .
$$


Clearly, for each $n \in \mathbb{N}$,

$$
C_{f, n}=\left\{(f(-, q), f(p,-)) \mid p, q \in \mathbb{Q}, f(p, q) \neq 0,0<q-p<\frac{1}{n}\right\}
$$

is a strong paircover of the sublocale lattice $(\mathcal{S}(L), \mathfrak{c} L, \mathfrak{o} L)$. Further, we have [6]:

Lemma 3.4. (1) For any $f_{1}, \ldots, f_{k} \in \operatorname{USC}(L), n_{1}, \ldots, n_{k} \in \mathbb{N}$ and $S \in$ $\mathcal{S}(L)$ :
(a) $s t_{1}\left(S, \bigwedge_{i=1}^{k} C_{f_{i}, n_{i}}\right) \in \mathfrak{c} L$.
(b) $s t_{2}\left(S, \bigwedge_{i=1}^{k} C_{f_{i}, n_{i}}\right) \in \mathfrak{o} L$.

(2) For any $a \in L$ and $n \in \mathbb{N}$ :

(a) $s t_{1}\left(\mathfrak{c}(a), C_{\chi_{\mathfrak{c}(a), n}}\right)=\mathfrak{c}(a)$.

(b) $s t_{2}\left(\mathfrak{o}(a), C_{\chi_{\mathfrak{c}(a), n}}\right)=\mathfrak{o}(a)$.

We have finally the required result that extends Proposition 1.1 of [14] (also Theorem 3.1 of [3]).

Proposition 3.5. $\left\{C_{f, n} \mid f \in \mathrm{USC}(L), n \in \mathbb{N}\right\}$ is a subbase for a quasiuniformity $\mathcal{U S C}(L)$ on the biframe $(\mathcal{S}(L), \mathfrak{c} L, \mathfrak{o} L)$.

Proof: For each $(f(-, q), f(p,-)) \in C_{f, 3 n}$ we have

$$
s t_{1}\left(f(-, q), C_{f, 3 n}\right) \leq f\left(-, q+\frac{1}{3 n}\right)
$$

and

$$
s t_{2}\left(f(p,-), C_{f, 3 n}\right) \leq f\left(p-\frac{1}{3 n},-\right)
$$

(the proof goes as in Proposition 3.2). Since $f\left(p-\frac{1}{3 n}, q+\frac{1}{3 n}\right) \geq f(p, q) \neq 0$, this shows that $C_{f, 3 n}^{*} \leq C_{f, n}$.

Conditions (U1) and (U3) follow immediately from Lemma 3.4.

$\mathcal{U S C}(L)$ is called the semicontinuous quasi-uniformity on $L$. This can be immediately generalized to any collection $\mathcal{C}$ containing all characteristic functions $\chi_{S}$ for a closed sublocale $S$ :

Corollary 3.6. Let $\mathcal{C}$ be a collection of upper semicontinuous real functions, containing all upper characteristic functions $\chi_{\mathfrak{c}(a)}(a \in L)$. Then $\left\{C_{f, n} \mid f \in \mathcal{C}, n \in \mathbb{N}\right\}$ is a subbase for a quasi-uniformity $\mathcal{U}_{\mathcal{C}}$ on the biframe $(\mathcal{S}(L), \mathfrak{c} L, \mathfrak{o} L)$. 


\section{Properties of $\mathcal{U S C}(L)$}

Proposition 4.1. $\mathcal{U S C}(L)$ is the coarsest quasi-uniformity $\mathcal{U}$ on $(\mathcal{S}(L), \mathfrak{c} L, \mathfrak{o} L)$ for which each biframe map $h:\left(\mathfrak{L}(\mathbb{R}), \mathfrak{L}_{l}(\mathbb{R}), \mathfrak{L}_{u}(\mathbb{R})\right) \rightarrow(\mathcal{S}(L), \mathfrak{c} L, \mathfrak{o} L)$ is a uniform homomorphism $h:\left(\left(\mathfrak{L}(\mathbb{R}), \mathfrak{L}_{l}(\mathbb{R}), \mathfrak{L}_{u}(\mathbb{R})\right), \mathcal{Q}\right) \rightarrow((\mathcal{S}(L), \mathfrak{c} L, \mathfrak{o} L), \mathcal{U})$.

Proof: We begin by checking that any biframe map

$$
h:\left(\mathfrak{L}(\mathbb{R}), \mathfrak{L}_{l}(\mathbb{R}), \mathfrak{L}_{u}(\mathbb{R})\right) \rightarrow(\mathcal{S}(L), \mathfrak{c} L, \mathfrak{o} L)
$$

is a uniform homomorphism

$$
\left(\left(\mathfrak{L}(\mathbb{R}), \mathfrak{L}_{l}(\mathbb{R}), \mathfrak{L}_{u}(\mathbb{R})\right), \mathcal{Q}\right) \rightarrow((\mathcal{S}(L), \mathfrak{c} L, \mathfrak{o} L), \mathcal{U S C}(L)),
$$

that is, $h\left[Q_{n}\right] \in \mathcal{U S C}(L)$ for every $n \in \mathbb{N}$. Obviously, the frame map $h$ : $\mathfrak{L}(\mathbb{R}) \rightarrow \mathcal{S}(L)$ belongs to $\operatorname{USC}(L)$. It suffices then to show that $C_{h, n} \leq h\left[Q_{n}\right]$, which is obvious since $C_{h, n} \subseteq h\left[Q_{n}\right]$.

Now let $\mathcal{U}$ be a quasi-uniformity on $(\mathcal{S}(L), \mathfrak{c} L, \mathfrak{o} L)$ for which any biframe map

$$
h:\left(\mathfrak{L}(\mathbb{R}), \mathfrak{L}_{l}(\mathbb{R}), \mathfrak{L}_{u}(\mathbb{R})\right) \rightarrow(\mathcal{S}(L), \mathfrak{c} L, \mathfrak{o} L)
$$

is a uniform homomorphism

$$
h:\left(\left(\mathfrak{L}(\mathbb{R}), \mathfrak{L}_{l}(\mathbb{R}), \mathfrak{L}_{u}(\mathbb{R})\right), \mathcal{Q}\right) \rightarrow((\mathcal{S}(L), \mathfrak{c} L, \mathfrak{o} L), \mathcal{U}) .
$$

In order to show that $\mathcal{U S C}(L) \subseteq \mathcal{U}$ it suffices to check that, for any $f \in$ $\operatorname{USC}(L)$ and $n \in \mathbb{N}, C_{f, n} \in \mathcal{U}$. By hypothesis,

$$
f\left[Q_{n}\right]=\left\{(f(-, q), f(p,-)) \mid p, q \in \mathbb{Q}, 0<q-p<\frac{1}{n}\right\} \in \mathcal{U} .
$$

So there is a strong paircover $C \in \mathcal{U}$ such that $C \leq f\left[Q_{n}\right]$. Then $C \leq C_{f, n}$. Indeed, for any $((-, q),(p,-)) \in C$ there are $\tilde{p}, \tilde{q} \in \mathbb{Q}$ with $(-, q) \leq f(-, \tilde{q})$, $(p,-) \leq f(\tilde{p},-)$ and $0<\tilde{q}-\tilde{p}<\frac{1}{n}$; since $(p, q) \neq 0$, then $f(\tilde{p}, \tilde{q}) \neq 0$.

Hence $C_{f, n} \in \mathcal{U}$ as required.

For every frame $L$,

$$
\{(\mathfrak{c}(a), 1),(1, \mathfrak{o}(a)) \mid a \in L\}
$$

is a subbase for a quasi-uniformity on $(\mathcal{S}(L), \mathfrak{c} L, \mathfrak{o} L)$ [8]. It is clearly a quasiuniformity compatible with the given frame $L$ since the first subframe $c L$ is an isomorphic copy of $L$. This is the pointfree analogue of the Császár-Pervin quasi-uniformity of a set $X$. We refer to it as the Frith quasi-uniformity and denote it by $\mathcal{F}$. 
Since

$$
C_{\chi_{\mathfrak{c}(a)}, n}=\left\{\left(\chi_{\mathfrak{c}(a)}(-, q), \chi_{\mathfrak{c}(a)}(p,-)\right) \mid p, q \in \mathbb{Q}, 0<q-p<\frac{1}{n}, \chi_{\mathfrak{c}(a)}(p, q) \neq 0\right\},
$$

then it is straightforward to check the following.

Lemma 4.2. For each characteristic function $\chi_{\mathfrak{c}(a)}, a \in L$,

$$
C_{\chi_{\mathfrak{c}(a)}, n}=\{(\mathfrak{c}(a), 1),(1, \mathfrak{o}(a))\} .
$$

Therefore, for $\mathcal{C}=\left\{\chi_{\mathfrak{c}(a)} \mid a \in L\right\}, \mathcal{U}_{\mathcal{C}}$ and $\mathcal{F}$ have a common subbase and we have:

Corollary 4.3. Let $\mathcal{C}=\left\{\chi_{\mathfrak{c}(a)} \mid a \in L\right\}$. Then $\mathcal{U}_{\mathcal{C}}=\mathcal{F}$.

A real-valued function $f \in \mathrm{F}(L)$ is bounded [12] if there exist some $p<q$ in $\mathbb{Q}$ for which $f(p, q)=1$. More generally, $f$ is upper bounded if $f(-, q)=1$ for some $q \in \mathbb{Q}$. Since every upper characteristic function $\chi_{\mathfrak{c}(a)}$ is bounded, the previous corollary leads immediately to the following result, which is the pointfree extension of Proposition 2.10 of [7].

Proposition 4.4. Let $\mathcal{C}$ be the collection of all bounded upper semicontinuous real functions on $L$. Then $\left\{C_{f, n} \mid f \in \mathcal{C}, n \in \mathbb{N}\right\}$ is a subbase for $\mathcal{F}$.

Proposition 4.5. Let $h:(\mathcal{S}(L), \mathfrak{c} L, \mathfrak{o} L) \rightarrow(\mathcal{S}(M), \mathfrak{c} M, \mathfrak{o} M)$ be a biframe map. Then $h$ is a uniform homomorphism

$$
((\mathcal{S}(L), \mathfrak{c} L, \mathfrak{o} L), \mathcal{U S C}(L)) \rightarrow((\mathcal{S}(M), \mathfrak{c} M, \mathfrak{o} M), \mathcal{U S C}(M)) .
$$

Proof: Let $C_{f, n} \in \mathcal{U S C}(L)$, for some $f \in \operatorname{USC}(L)$ and $n \in \mathbb{N}$. Evidently, $h f \in \operatorname{USC}(M)$ and

$$
\begin{aligned}
h\left[C_{f, n}\right] & =\left\{(h f(-, q), h f(p,-)) \mid p, q \in \mathbb{Q}, 0<q-p<\frac{1}{n}, f(p, q) \neq 0\right\} \\
& \geq C_{h f, n} \in \mathcal{S} C(M)
\end{aligned}
$$

because $h f(p, q) \neq 0 \Rightarrow f(p, q) \neq 0$.

We say that a quasi-uniform biframe $(L, \mathcal{U})$ is totally bounded if $\mathcal{U}$ has a base of finite paircovers.

Lemma 4.6. If $((\mathcal{S}(L), \mathfrak{c} L, \mathfrak{o} L), \mathcal{U})$ is a totally bounded quasi-uniform biframe then every uniform homomorphism

$$
h:\left(\left(\mathfrak{L}(\mathbb{R}), \mathfrak{L}_{l}(\mathbb{R}), \mathfrak{L}_{u}(\mathbb{R})\right), \mathcal{Q}\right) \rightarrow((\mathcal{S}(L), \mathfrak{c} L, \mathfrak{o} L), \mathcal{U})
$$

is bounded. 
Proof: Let $h:\left(\left(\mathfrak{L}(\mathbb{R}), \mathfrak{L}_{l}(\mathbb{R}), \mathfrak{L}_{u}(\mathbb{R})\right), \mathcal{Q}\right) \rightarrow((\mathcal{S}(L), \mathfrak{c} L, \mathfrak{o} L), \mathcal{U})$ be a uniform homomorphism. For each $n \in \mathbb{N}, h\left[Q_{n}\right] \in \mathcal{U}$, so there exists a finite paircover

$$
C=\left\{\left(\mathfrak{c}\left(a_{1}\right), \mathfrak{o}\left(b_{1}\right)\right), \cdots,\left(\mathfrak{c}\left(a_{k}\right), \mathfrak{o}\left(b_{k}\right)\right)\right\}
$$

of $\mathcal{S}(L)$ such that $C \leq h\left[Q_{n}\right]$. Therefore, for each $i \in\{1, \cdots, k\}, \mathfrak{c}\left(a_{i}\right) \leq$ $h\left(-, q_{i}\right)$ and $\mathfrak{o}\left(b_{i}\right) \leq h\left(p_{i},-\right)$ for some $p_{i}, q_{i} \in \mathbb{Q}$ with $0<q_{i}-p_{i}<\frac{1}{n}$. Hence $1=\bigvee_{i=1}^{k} \mathfrak{c}\left(a_{i}\right) \wedge \mathfrak{o}\left(b_{i}\right) \leq \bigvee_{i=1}^{k} h\left(p_{i}, q_{i}\right)$. Let $q=\max _{i=1, \ldots, k} q_{i}$ and $p=\min _{i=1, \ldots, k} p_{i}$. Immediately, $h(p, q)=1$ and $h$ is bounded.

Proposition 4.7. Let $((\mathcal{S}(L), \mathfrak{c} L, \mathfrak{o} L), \mathcal{U})$ be a totally bounded quasi-uniform frame. Then there exists a collection $\mathcal{C}$ of bounded $f \in \operatorname{USC}(L)$ such that $\left\{C_{f, n} \mid f \in \mathcal{C}, n \in \mathbb{N}\right\}$ is a subbase for $\mathcal{U}$.

Proof: Let $((\mathcal{S}(L), \mathfrak{c} L, \mathfrak{o} L), \mathcal{U})$ be a totally bounded quasi-uniform frame. Every uniform homomorphism

$$
h:\left(\left(\mathfrak{L}(\mathbb{R}), \mathfrak{L}_{l}(\mathbb{R}), \mathfrak{L}_{u}(\mathbb{R})\right), \mathcal{Q}\right) \rightarrow((\mathcal{S}(L), \mathfrak{c} L, \mathfrak{o} L), \mathcal{U}),
$$

which is bounded by Lemma 4.6, is upper semicontinuous. Let $\mathcal{C}$ be the collection of every such maps. Since $\mathcal{C}$ contains all characteristic functions $\chi_{\mathfrak{c}(a)}(a \in L)$, then, by Corollary 3.6, $\left\{C_{h, n} \mid h \in \mathcal{C}, n \in \mathbb{N}\right\}$ is a subbase for a quasi-uniformity $\mathcal{U}_{\mathcal{C}}$ on $(\mathcal{S}(L), \mathfrak{c} L, \mathfrak{o} L)$. Since $h$ is uniform,

$$
h\left[Q_{n}\right]=\left\{(h(-, q), h(p,-)) \mid p, q \in \mathbb{Q}, 0<q-p<\frac{1}{n}\right\} \in \mathcal{U} .
$$

So there is a strong paircover $C \in \mathcal{U}$ such that $C \leq h\left[Q_{n}\right]$. Then $C \leq C_{h, n}$ (the proof is similar to the proof at the end of 4.1 that $C \leq C_{f, n}$ ). Hence $\left\{C_{h, n} \mid h \in \mathcal{C}, n \in \mathbb{N}\right\}$ is also a subbase for $\mathcal{U}$.

Theorem 4.8. Let $L$ be a frame. Then $\mathcal{U S C}(L)$ is totally bounded if and only if every $f \in \operatorname{USC}(L)$ is bounded.

Proof: Assume that $\mathcal{U S C}(L)$ is totally bounded and let $f \in \operatorname{USC}(L)$. Then we have a biframe map $f:\left(\mathfrak{L}(\mathbb{R}), \mathfrak{L}_{l}(\mathbb{R}), \mathfrak{L}_{u}(\mathbb{R})\right) \rightarrow(\mathcal{S}(L), \mathfrak{c} L, \mathfrak{o} L)$ which, by Proposition 4.1, is uniform. Then, by Lemma 4.6, $f$ is bounded.

Conversely, let $\mathcal{C}=\operatorname{USC}(L)=\{$ bounded $f \in \operatorname{USC}(L)\}$. Then $\mathcal{U}_{\mathcal{C}}=$ $\mathcal{U S C}(L)$ coincides by Proposition 4.4 with $\mathcal{F}$. Since $\mathcal{F}$ is totally bounded, then $\mathcal{U S C}(L)$ is totally bounded.

Recall that a frame is countably compact if each countable cover has a finite subcover. 
Theorem 4.9. Let $L$ be a frame. Then every $f \in \operatorname{USC}(L)$ is upper bounded if and only if $L$ is countably compact.

Proof: Let $A=\left\{a_{i} \mid i \in \mathbb{N}\right\}$ be a countable cover of $L$. For each $q \in \mathbb{Q}$ let $m(q)=\min \left\{n \in \mathbb{N}_{0} \mid n \geq q\right\}$. Further, let $a_{0}=0$ and define, for each $r \in \mathbb{Q}$,

$$
S_{r}=\mathfrak{o}\left(\bigvee_{i=0}^{m(r)} a_{i}\right)
$$

This is clearly a scale of open sublocales so, by (2.1), the function $f$ defined by

$$
f(p,-)=\bigvee_{r>p} \mathfrak{o}\left(\bigvee_{i=0}^{m(r)} a_{i}\right) \quad \text { and } \quad f(-, q)=\bigvee_{r<q} \mathfrak{c}\left(\bigvee_{i=0}^{m(r)} a_{i}\right) \quad(p, q \in \mathbb{Q})
$$

is in $\operatorname{USC}(L)$. By hypothesis, $f$ is bounded. Consequently, there is some $q \in \mathbb{Q}$ for which $f(-, q)=1$. This means precisely that

$$
1=\bigvee_{r<q} \mathfrak{c}\left(\bigvee_{i=0}^{m(r)} a_{i}\right)=\mathfrak{c}\left(\bigvee_{r<q} \bigvee_{i=0}^{m(r)} a_{i}\right)=\mathfrak{c}\left(\bigvee_{i=0}^{m(q)} a_{i}\right)
$$

that is, $\bigvee_{i=0}^{m(q)} a_{i}=1$. Hence $\left\{a_{1}, \ldots, a_{m(q)}\right\}$ is a finite subcover of $A$. This shows that $L$ is countably compact.

Conversely, let $L$ be countably compact and let $f \in \operatorname{USC}(L)$. Then $\{f(-, q) \mid q \in \mathbb{Q}\}$ is a countable cover of $\mathfrak{c} L \cong L$. By hypothesis, there exist $q_{1}, \ldots, q_{k} \in \mathbb{Q}$ such that $\bigvee_{i=1}^{k} f\left(-, q_{i}\right)=1$, that is, $f\left(-, \bigvee_{i=1}^{k} q_{i}\right)=1$, which shows that $f$ is upper bounded.

This is the pointfree counterpart of Lemma 3.2 of [3]. Our last result extends Corollary 3.3 of [3]. It asserts that every frame $L$ with a unique compatible quasi-uniform structure is countably compact.

Corollary 4.10. If $(\mathcal{S}(L), \mathfrak{c} L, \mathfrak{o} L)$ has a unique quasi-uniform structure then $L$ is countably compact.

Proof: If $\mathcal{U}$ is the unique quasi-uniform structure on $(\mathcal{S}(L), \mathfrak{c} L, \mathfrak{o} L)$ then $\mathcal{U}$ coincides with $\mathcal{F}$ which is totally bounded. But also $\mathcal{U}=\mathcal{U S C}(L)$ so, by the theorems above, $L$ is countably compact. 


\section{References}

[1] B. Banaschewski, The Real Numbers in Pointfree Topology, Textos de Matemática, Vol. 12, University of Coimbra, 1997.

[2] B. Banaschewski, G.C. L. Brümmer and K. A. Hardie, Biframes and bispaces, Quaest. Math. 6 (1983) 13-25.

[3] C. Barnhill and P. Fletcher, Topological spaces with a unique compatible quasi-uniform structure, Arch. Math. (Basel) 21 (1970) 206-209.

[4] M. J. Ferreira, Sobre a construção de estruturas quase-uniformes em Topologia sem Pontos, $\mathrm{PhD}$ thesis, University of Coimbra, 2004.

[5] M. J. Ferreira and J. Picado, Functorial quasi-uniformities on frames, Appl. Categ. Structures 13 (2005) 281-303.

[6] M. J. Ferreira and J. Picado, The semicontinuous quasi-uniformity of a frame, Kyungpook Math. J. 46 (2006) 299-306.

[7] P. Fletcher and W.F. Lindgren, Quasi-uniform Spaces, Marcel Dekker, New York, 1982.

[8] J. Frith, Structured frames, PhD thesis, University of Cape Town, 1987.

[9] J. Frith, The category of quasi-uniform frames, Research Reports, Department of Mathematics, University of Cape Town, 1991.

[10] J. Frith and A. Schauerte, The Samuel compactification for quasi-uniform biframes, Topol. Appl. 156 (2009) 2116-2122.

[11] J. Gutiérrez García, T. Kubiak and J. Picado, Lower and upper regularizations of frame semicontinuous real functions, Algebra Univ. 60 (2009) 169-184.

[12] J. Gutiérrez García, T. Kubiak and J. Picado, Localic real functions: a general setting, J. Pure Appl. Algebra 213 (2009) 1064-1074.

[13] P. T. Johnstone, Stone Spaces, Cambridge Studies in Advanced Mathematics, Vol. 3, Cambridge University Press, Cambridge, 1982.

[14] R. Nielsen and C. Sloyer, Quasi-uniformizability, Math. Ann. 182 (1969) 273-274.

[15] J. Picado, Weil uniformities for frames, Comment. Math. Univ. Carolin. 36 (1995) 357-370.

[16] J. Picado, Frame quasi-uniformities by entourages, in: Symposium on Categorical Topology (University of Cape Town 1994), Department of Mathematics, University of Cape Town, 1999, pp. 161-175.

[17] J. Picado, Structured frames by Weil entourages, Appl. Categ. Structures 8 (2000) 351-366.

[18] J. Picado and A. Pultr, Locales Mostly Treated in a Covariant Way, Textos de Matemática, Vol. 41, University of Coimbra, 2008.

MARIA Jõ̃o FERREIRA

CMUC, Department of Mathematics, University of Coimbra, 3001-454 Coimbra, Portugal

E-mail address: mjrf@mat.uc.pt

JORGE PICADO

CMUC, Department of Mathematics, University of Coimbra, 3001-454 Coimbra, Portugal

E-mail address: picado@mat.uc.pt 